\title{
Optical coherence tomography angiography - novel method to access retinal microcirculation in patients with diabetic retinopathy
}

\section{ABSTRACT}

Introduction. Optical coherence tomography angiography (OCTA) is a novel technique that allows visualization of retinal capillary network without the need for contrast usage. Being easy to perform and non-invasive, it might become significantly competitive to fluoroangiography.

Material and methods. The study included 45 patients (90 eyes) with diabetes type 1 and 2 and nonproliferative and proliferative diabetic retinopathy as well as 35 healthy volunteers (70 eyes) as a control group. Beside full ophthalmic examination, all patients underwent OCTA (RTVue XR Avanti; Optovue) examination. Obtained images were used to assess the presence of morphologic changes of the retina characteristic for diabetic retinopathy (capillary density and regularity as well as occurrence of microaneurysms, ischemic areas, intraretinal microvascular abnormalities and neovascularizations). The assessment was carried out on two levels of retinal capillary plexus. Additionally, measurement of foveal avascular zone (FAZ) was performed. The results were compared with those obtained from the control group.

Address for correspondence:

lek. Małgorzata Loba

Klinika Chorób Wewnętrznych i Diabetologii

Uniwersytet Medyczny w Łodzi

ul. Pomorska 251, 92-013 Łódź

Phone/fax: +48 (42) 2014340

e-mail: gosialoba@onet.pl

Translation: dr med. Jędrzej Toczko,

dr hab. med. Anna Korzon-Burakowska

Clinical Diabetology 2015, 5, 3, 77-82

DOI: $10.5603 /$ DK.2016.0013

Received: 25.01.2016

Accepted: 15.04.2016
Results. All examined eyes in the studied group exhibited one or more anomalies characteristic of diabetic retinopathy as seen on the OCTA scans, both in superficial and deep vascular plexus. In the control group we have detected capillary irregularity ( $9 \%$ in a superficial and $11 \%$ in a deep vascular plexus) and capillary rarefication (7\% in a superficial and $9 \%$ in a deep vascular plexus) and no other anomalies were found. Mean FAZ area measured in superficial vascular plexus was $382 \pm$ \pm 134 square micrometers and was significantly larger $(p<0,001)$ in comparison to the control group (259 \pm \pm 103 square micrometers).

Conclusions. Optical coherence tomography angiography is a useful diagnostic tool used to assess vascular changes in the retinal microcirculation in diabetic patients. Its main advantages are noninvasiveness, repeatability and a high quality of images acquired from the chosen level of the retina. (Clin Diabet 2016; 5, 3: 77-82)

Key words: diabetic retinopathy, angiography, optical coherence tomography

\section{Introduction}

Diabetes is oftentimes vividly defined as a disease of blood vessels. The negative influence of chronic, drawn-out hyperglycemia on microcirculation is well documented in literature reports [1]. Blood flow disturbance in small caliber vessels associated with permanent hyperglycemia affects all organs, but is easiest to observe within the eye. This is because vitreous body is transparent and, with adequate optical equipment, we can effortlessly and precisely assess the degree of vascular changes in the fundus of the eye. This fact 
has been known for years, although ongoing technological progress gives us access to unprecedented possibilities [2].

Retinopathy is a relatively common complication of diabetes. In screening studies conducted amongst patients with at least 5 years history of diabetes, over $50 \%$ exhibit changes in the fundus of the eye typical for the disease [3]. Although a significant progression of retinopathy occurs only in a relatively small group of patients, we are not able to predict who will be affected by it. Because of this a full ophthalmic examination is periodically performed in all patients with changes in the fundus of the eye [4]. Postponing the onset of laser therapy is a mistake, as this method of treatment is the only effective way of saving vision in diabetic retinopathy. Thus, the cost of monitoring progression of retinal vascular changes in diabetic patients is significant and there is much need for simplification of associated procedures, while maintaining their sensitivity and precision.

Every patient with diabetes - both type 1 and type 2 - should annually undergo an eye examination with thorough assessment of the fundus of the eye, performed after pupil dilatation [4]. Some patients present changes that require further investigation. Currently, the gold standard for retinal circulation assessment is fluorescein angiography (FA). It is an invasive and time consuming procedure. Furthermore, complications after intravenous application of fluorescein - however rare - can be threatening, e.g. anaphylactic shock. If these occur, further FA's are contraindicated in such patients. Consequently, the procedure is forfeited more often than it should be. This of course increases the risk of introducing the laser therapy too late.

For a couple of years now there has been intensive ongoing research concerning creation of new imaging methods for the fundus of the eye, without the need of using contrast. Techniques based on optical coherence tomography, which is currently at its golden age, proved to be most promising. Optical coherence tomography angiography (OCTA) is a method that operates on a basis of imaging the movement of erythrocytes in blood vessels, enabling a visualization of vascular network of both the retina and the choroid (Fig. 1). The examination takes from several to several dozen seconds and does not require pupil dilatation. It can be performed repeatedly without any threat to the patient.

\section{Study goal}

The goal of the study was to asses retinal microcirculation using the optical coherence tomography angiography in patients with diabetes and diabetic retinopathy in different progression stages.

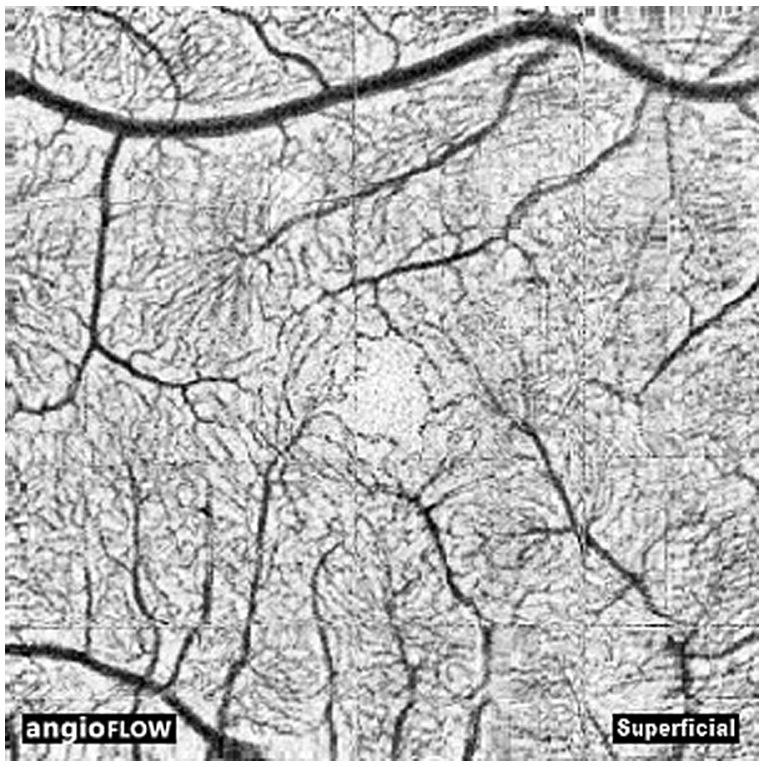

Figure 1. Example image of fovelar area obtained with OCTA method

\section{Material and methods}

The study was conducted in Diabetology Outpatient Clinic of N. Barlicki University Hospital No. 1 in Lodz from 23.02.2015 to 21.03.2015. Observations were performed in in accordance with the study protocol in all patients with type 1 and type 2 diabetes visiting the clinic consecutively, who met the inclusion criteria. Based on medical history and blood tests results from month prior to the start of the study, patients with $\mathrm{HbA}_{1 \mathrm{c}}>8.5 \%$, renal insufficiency, smokers and those previously treated with laser therapy or anti-VEGF, were excluded from the study.

Qualified patients underwent a full ophthalmic examination. Based on its results, the next phase of the study included patients with transparent visual pathway and clinical signs of diabetic retinopathy, both proliferative and non-proliferative.

A control group was formed from recruited volunteers, matched by gender and age, non smokers, without prior history of ophthalmologic treatment of any kind, with no abnormalities present in an ophthalmologic examination.

Eventually the study group (group 1) consisted of 45 patients (90 eyes), 22 men (49\%) and 23 women (51\%) aged from 31 to 82 (mean age: $64 \pm 13$ ). The control group (group 2) was formed by 35 persons (70 eyes) without diabetes, 19 women (54\%) and 15 men (46\%) aged from 35 to 75 (mean value $62 \pm 9$ ). Mean diabetes duration time in the study group stood at $11 \pm 7.4$ years.

Every person included in the study underwent an OCTA (RTVue XR Avanti; Optovue) examination. 
The device performed $70000 \mathrm{~A}$-scans per second on a $6 \times$ $\times 6 \mathrm{~mm}$ area surrounding the fovea, on two levels of deep and superficial retinal capillary plexus. Using the SSADA (Split-Spectrum Amplitude Decorrelation Angiography) algorithm, the machine detects red blood cells flow in order to visualize the capillary network. The image is then laid over a two dimensional map of the retina acquired with SSOCT (Swept-source Optical Coherence Tomography) in EN FACE 3D mode. The scanning procedure of one eye took 20 seconds on average. The examination was performed without pharmacological pupil dilatation.

Acquired angiographic images were assessed and compared in regard of sings that in classical FA examinations are characteristic of diabetic retinopathy. Disturbance in vascular architecture is the most prominent feature of diabetic retinopathy. Therefore, the regularity and density of the capillary network were assessed. In extreme cases, lack of capillaries indicates the presence of ischemic areas. Microaneurysms - bulges on the vascular wall - visible clinically as reddish points and angiographically as point-like, hyperfluorescent areas, are also typical for diabetes. Their numbers increase along with the progression of diabetic changes. Presence of intraretinal microvascular abnormalities (IRMA) — invalid arteriovenous anastomoses in low perfusion areas - and neovascularisations (NVE) - pathological, newly formed vessels - indicates progression to preproliverative and proliferative retinopathy respectively.

All parameters were assessed both on deep and superficial levels of the retinal plexus. The superficial plexus spreads from the inner limiting membrane to the inner plexiform layer which adds up to approximately $120 \mathrm{mi}-$ crons. This layer encompasses main retinal vessels, with capillaries decreasing in diameter towards the macula, creating concentric junctions that resemble a spider's web. Meanwhile, the deep plexus consists of the further 60 microns up to the outer plexiform layer. It includes lesser capillaries with multiple connections and similar diameter throughout the area, creating fanlike branches around the foveal avascular zone. Many anastamoses connect these two plexi, perpendicular to their surface.

Additionally, the surface of the foveal avascular zone (FAZ) was measured for the superficial layer using an Image J (NIMH, Bethesda, USA) graphic program. The FAZ is an area surrounding the fovea and completely deprived of vessels, it can only be properly assessed using angiographic images. Physiologically, its surface area peaks at approximately 270 square micrometers and may increase in some vascular diseases of the retina [5].

Statistical analysis was performed with the use of parametric and non-parametric tests. Calculations were carried out assuming $\alpha=0.05$ statistical significance level and using Microsoft Excel and Addinsoft XLStat 2008 software. This study was approved by the Bioethics Committee of the Medical University of Lodz (Dec nr. RNN/328/15/KE).

\section{Results}

All of the tested eyes of patients in the group 1 displayed one or more features characteristic for diabetic retinopathy, using the OCTA examination, on both retinal levels that were examined. In the group 2, none of the eyes displayed microaneurysms, ischemic areas, IRMA or NVE, however the capillary network was assessed as irregular - in $9 \%$ on superficial level and $11 \%$ on deep level - or rareficated - in $7 \%$ on superficial and $9 \%$ on deep level. Figure 2 shows an illustrative angiogram showing features characteristic of diabetic retinopathy in OCTA imaging.

Table 1 presents a summary of morphological features of the superficial and deep plexi. A comparison of morphological parameters of capillary networks between the groups of diabetic patients and the control group showed a statistically significant difference on both superficial and deep vascular plexus levels for every assessed parameter $(p<0.05)$.

The mean surface area of FAZ measured on the superficial level in the group 1 was $382 \pm 134$ square micrometers and was significantly larger than in the group 2, which presented a mean area of $259 \pm 103$ square micrometers. This correlations were statistically significant $(p<0.001)$. An example image of an enlarged FAZ in a patient with diabetic retinopathy in comparison to a healthy individual is presented in Figure 3.

\section{Discussion}

A novel angiographic technique based on optical coherence tomography is the next milestone in the vascular diseases of the retina diagnosing process. Its viability increases in patients who require numerous, repeatable examinations. This applies especially to patients with diabetic retinopathy, as its progression stage may require introducing laser therapy at any time. Results of this study prove that OCTA is capable of detecting and imaging vascular abnormalities in the retina characteristic of diabetic retinopathy. However it remains an open question, if it can be a serious competition to fluorescein angiography as the gold standard for diagnosing vascular diseases of the retina.

Previous studies comparing OCTA angiograms with classic FA images show many benefits of the former $[6,7]$. First of all, OCTA angiograms feature high resolution. Obtained images present an uncanny quality in 


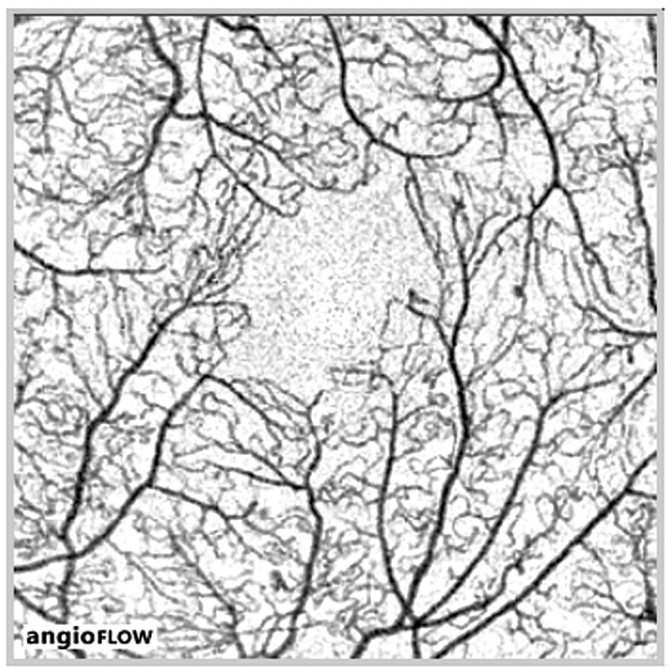

Angio / OCT - Superficial

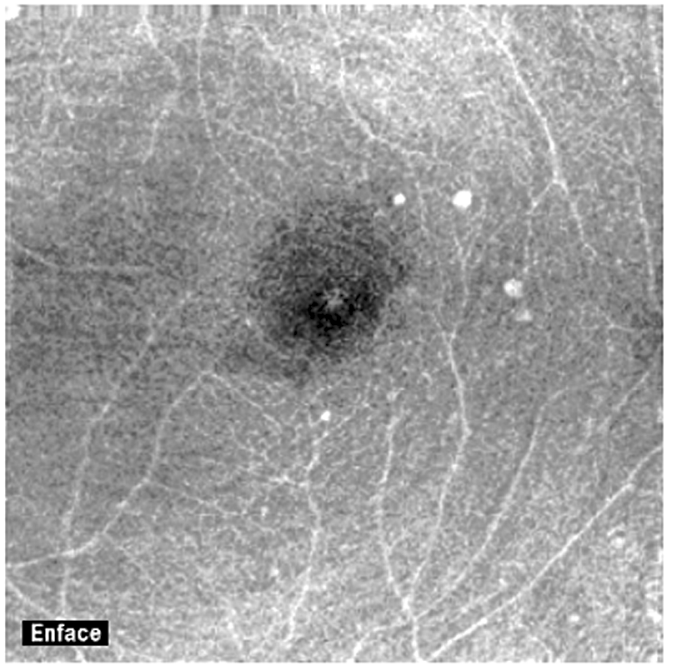

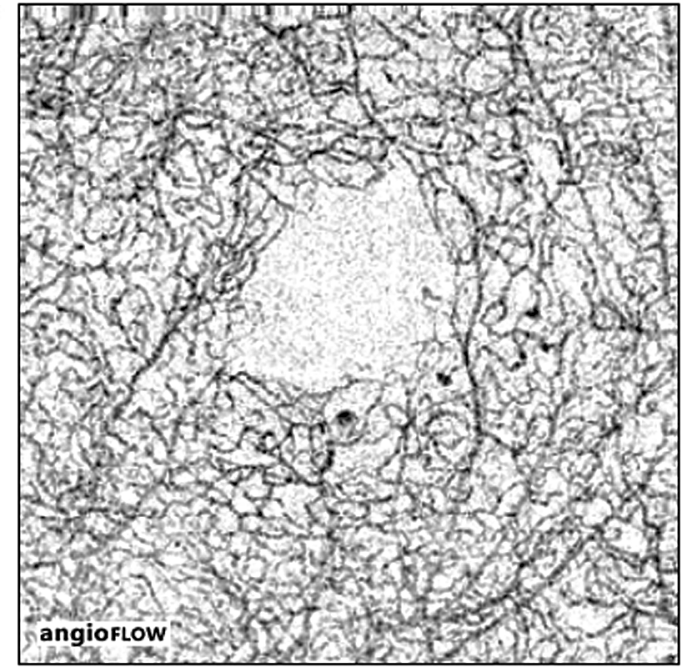

Angio / OCT - Deep

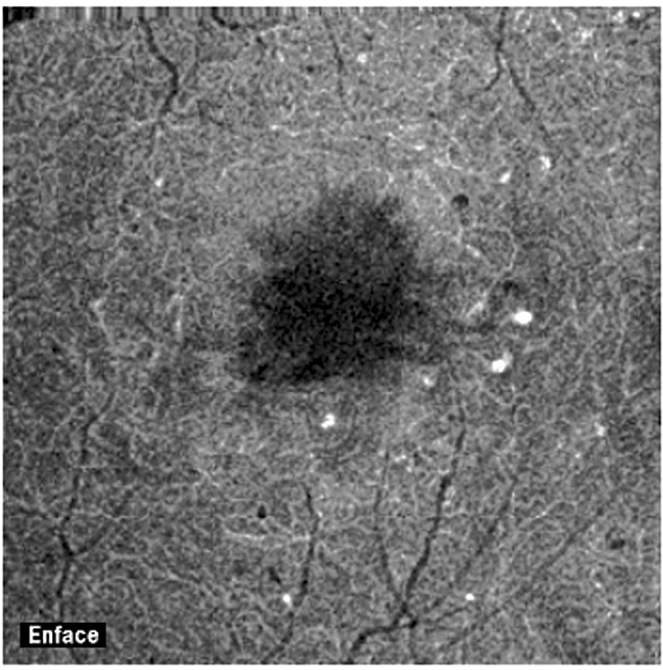

Figure 2. An angiogram showing characteristic features of diabetic retinopathy in OCTA imaging. The left side shows superficial layer images, with deep layer images on the right. Bottom scans present an angiographic image superimposed on top of an OCT en face scan

mapping the smallest capillary network surrounding the macula, which enables a visualization of minimal structural abnormalities [6, 8]. This could potentially lead to diagnosing vascular changes in diabetic patients much earlier than it is currently possible, but would also require performing OCTA on a large group of patients [9]. Another formidable advantage of OCTA when compared to FA is the ability to obtain images from various layers of the retina. This is a whole new quality, as it has thus been impossible to differentiate the deep and superficial vascular plexus in an image. Differences in progression of changes in these layers have not been thoroughly examined yet and their significance is still ambiguous, but they may serve as starting point to further studies on the diabetic retinopathy patomechanism.
However, some shortcomings of this new method should still be noted. FA holds an evident advantage in visualizing capillary leaks and microanuerysmal leakiness $[6,7]$. This is of significance because these signs determine the decision to introduce focal laser therapy or anti-VEGF treatment. Nonetheless, OCTA may greatly reduce the numbers of performed FA examinations, enabling easier access to FA for patients who absolutely need it.

A fundamental difference between OCTA and FA is the way in which the information regarding vascular blood flow is obtained. Fluoroscein angiography utilizes contrast in order to do so. This makes pathologies related to dye accumulation, such as microaneursyms, more visible. On the other hand, OCTA operates on detecting movement of blood cells - in other words, 
Table 1. A comparison of morphological features of the deep and superficial retinal vascular plexus in diabetic retinopathy patients (group 1) and healthy individuals (group 2)

\begin{tabular}{|c|c|c|c|c|c|c|c|c|}
\hline \multirow{3}{*}{$\begin{array}{l}\text { Morphological features } \\
\text { Capillary network structure }\end{array}$} & \multicolumn{4}{|c|}{ Superficial vascular plexus } & \multicolumn{4}{|c|}{ Deep vascular plexus } \\
\hline & \multicolumn{2}{|c|}{ Group 1} & \multicolumn{2}{|c|}{ Group 2} & \multirow{2}{*}{$\frac{\text { Group } 1}{n}$} & \multicolumn{3}{|c|}{ Group 2} \\
\hline & $\mathrm{n}$ & $\%$ & $\mathrm{n}$ & $\%$ & & $\%$ & $\mathrm{n}$ & $\%$ \\
\hline Regular & 31 & 34 & 64 & 91 & 32 & 36 & 62 & 89 \\
\hline Irregular & 59 & 66 & 6 & 9 & 58 & 64 & 8 & 11 \\
\hline \multicolumn{9}{|l|}{ Density of capillary network } \\
\hline Dense & 15 & 16 & 65 & 93 & 12 & 13 & 64 & 91 \\
\hline Moderately dense & 34 & 38 & 5 & 7 & 26 & 29 & 6 & 9 \\
\hline Rarificated & 41 & 45 & 0 & 0 & 52 & 58 & 0 & 0 \\
\hline \multicolumn{9}{|l|}{ Presence of microaneurysms } \\
\hline None & 5 & 6 & 70 & 100 & 14 & 16 & 70 & 100 \\
\hline Singular & 33 & 37 & 0 & 0 & 34 & 38 & 0 & 0 \\
\hline Multiple & 52 & 58 & 0 & 0 & 42 & 47 & 0 & 0 \\
\hline \multicolumn{9}{|l|}{ Ischemic areas } \\
\hline None & 65 & 72 & 70 & 100 & 52 & 58 & 70 & 100 \\
\hline Singular & 18 & 20 & 0 & 0 & 27 & 30 & 0 & 0 \\
\hline Multiple & 7 & 8 & 0 & 0 & 11 & 12 & 0 & 0 \\
\hline \multicolumn{9}{|l|}{ IRMA } \\
\hline None & 31 & 34 & 70 & 100 & 24 & 27 & 70 & 100 \\
\hline Singular & 51 & 57 & 0 & 0 & 58 & 64 & 0 & 0 \\
\hline Multiple & 8 & 9 & 0 & 0 & 8 & 9 & 0 & 0 \\
\hline \multicolumn{9}{|l|}{ Neovascularisations } \\
\hline None & 50 & 56 & 70 & 100 & 44 & 49 & 70 & 100 \\
\hline Singular & 35 & 39 & 0 & 0 & 39 & 43 & 0 & 0 \\
\hline Multiple & 5 & 6 & 0 & 0 & 7 & 8 & 0 & 0 \\
\hline
\end{tabular}

IRMA - intraretinal microvascular abnormalities
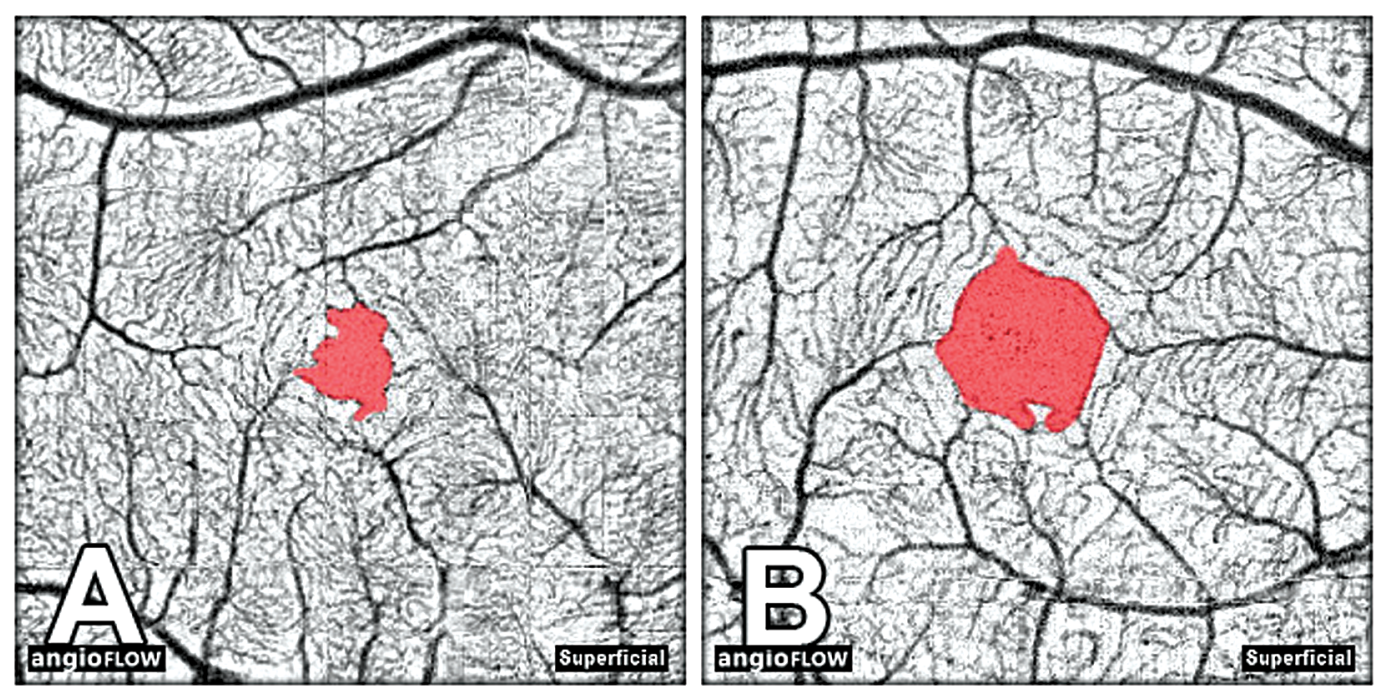

Figure 3. Example image of foveal avascular zone (FAZ) - in red - in a healthy individual (A) and an enlarged one in a patient with diabetic retinopathy $(B)$ 
an active blood flow - which results in the inability to detect leaking or congestion. However, this may be both beneficial and disadvantageous. While contrast accumulation in FA causes some details behind the given area to be obscured, there is no such problem in OCTA, as visualization of vascular changes is presented separately for each level.

The results of this study seem to confirm these assumptions. In regard of the superficial layer there were $43 \%$ of patients and in regard of the deep layer $54 \%$ of patients in the group 1 that showed only isolated or none at all microaneurysms, while clinical assessment disclosed them in every patient in this group. The reason for this discrepancy may be the low blood flow through the microaneurysms or flow of the serum without morphotic elements, which makes detecting them with decorelation method impossible. Numbers of microanerusyms lower than expected while using OCTA examinations were also reported by other authors $[6,8,9]$.

In contrast to FA, a relatively small surface area of the examination $(3 \times 3 \mathrm{~mm}$ or $6 \times 6 \mathrm{~mm})$ is a serious limitation of OCTA. This is due to problems of technical nature. However, there are works in progress aimed to increase the area accessible to the method, which in the near future may also enable covering peripheral parts of the fundus of the eye. It should be noted that patients with reduced transparency of the optical pathway for instance, with cataracts - won't be able to fully benefit from this method.

Retinal microcirculation assessment is non-parametric in both OCTA and FA examinations. Capillary rarerifcation or irregularity was present in $10 \%$ of patients from the control group on both capillary plexi levels. With OCTA providing an image so detailed showing the tiniest of capillaries - some range of anatomical variations is expected to be present, as it had not been described yet due to novelty of the method.

FAZ surface area is an important feature that can be easily measured and assessed using the OCTA method. This study disclosed a significant expansion of this area in patients with diabetic retinopathy. Other studies using the FA method confirmed that the increase in FAZ surface is a factor significantly correlated with progression of diabetic retinopathy and can be used to ascertain its progression level. However, using FA seemed too burdening at the time, because of the invasive nature of the procedure. Currently, OCTA enables a regular FAZ assessment in diabetic patients. Further studies are necessary to evaluate the utility of this parameter in assessment of diabetic retinopathy progression $[10,11]$.

In conclusion, it is worth noting that the condition of retinal and choroidal circulation can be an important indicator of general state of blood vessels of the individual.
Thus, being relatively easy to perform and non-invasive, OCTA may become a valuable procedure in systemic diseases such as atherosclerosis, hypertension, cerebral stroke, renal diseases or other vascular diseases [12]

\section{Conclusions}

OCTA is a useful diagnostic tool used to assess vascular changes in the retinal microcirculation in diabetic patients. Its main advantages are noninvasiveness, repeatability and a high quality of images acquired from the chosen level of the retina.

OCTA provides a detailed imaging of foveal avascular zone and can confirm the presence of features characteristic of diabetic retinopathy, such as ischemic areas, IRMA or NVE. Despite its limitations, it may become an important additional tool in diagnosing diabetic retinopathy.

\section{Conflict of interest}

No conflict of interest is declared by the authors.

\section{Acknowledgments}

Authors would like to thank Medical Partner Private Limited Company for lending the RTVue XR Avanti device by Optovue.

\section{REFERENCES}

1. The Diabetes Control and Complications Trial Research Group. The Effect of Intensive Treatment of Diabetes on the Development and Progression of Long-Term Complications in Insulin-Dependent Diabetes Mellitus. N Engl J Med 1993; 329: 977-986.

2. Hutchinson A, McIntosh A, Peters J et al. Effectiveness of screening and monitoring tests for diabetic retinopathy a systematic review. Diabet Med 2000; 17: 495-506.

3. Klein R, Klein BE, Moss SE, Cruickshanks KJ. The Wisconsin Epidemiologic Study of diabetic retinopathy. XIV. Ten-year incidence and progression of diabetic retinopathy. Arch Ophthalmol 1994; 112: 1217-1228.

4. Zalecenia kliniczne dotyczące postępowania u chorych na cukrzycę 2015, stanowisko PTD. Diabetologia Kliniczna 2015; 4: suppl A.

5. Conrath J, Giorgi R, Raccah D, Ridings B. Foveal avascular zone in diabetic retinopathy: quantitative vs qualitative assessment. Eye 2005; 19: 322-326.

6. Hwang TS, Jia Y, Gao SS et al. Optical coherence tomography angiography features of diabetic retinopathy. Retina 2015; 35: 2371-2376.

7. Couturier A, Mané V, Bonnin S et al. Capillary plexus anomalies in diabetic retinopathy on optical coherence tomography angiography. Retina 2015; 35: 2384-2391.

8. Ishibazawa A, Nagaoka T, Takahashi A et al. Optical Coherence Tomography Angiography in Diabetic Retinopathy: A Prospective Pilot Study. Am J Ophthalmol 2015; 160: 35-44.

9. de Carlo TE, Chin AT, Bonini Filho MA et al. Detection of microvascular changes in eyes of patients with diabetes but not clinical diabetic retinopathy using optical coherence tomography angiography. Retina 2015; 35: 2364-2370.

10. Takase N, Nozaki M, Kato A, Ozeki H, Yoshida M, Ogura Y. Enlargement of foveal avascular zone in diabetic eyes evaluated by en face optical coherence tomography angiography. Retina 2015; 35: 2377-2383.

11. Mansour AM, Schachat A, Bodiford G, Haymond R. Fovealavascular zone in diabetes mellitus. Retina 1993; 13: 125-128.

12. Wong TY, Mclntosh R. Systemic associations of retinal microvascular signs: a review of recent population-based studies. Ophthalmic Physiol Opt 2005; 25: 195-204. 